\title{
Arbor
}

\section{Lesiones en el deporte}

\section{Silvio Rubio Gimeno y Manuel Chamorro}

Arbor CLXV, 650 (Febrero 2000), 203-225 pp.

El incremento de la áctividad física y del deporte, en las sociedades llamadas desarrolladas, ha traído consigo beneficios claros para la salud, reflejados en diferentes indicadores de salud. Simultáneamente, el deporte de competición obliga a una dedicación diaria a intensidad de entrenamiento, con objeto de obtener los elevados requerimientos físicos que exige la competición. Todo ello ha traído consigo la aparición de numerosas lesiones, fundamentalmente del sistema músculo-esquelético.

Se exponen en este trabajo consideraciones históricas, la epidemiología de la lesión deportiva y se describen, concisamente, algunas de las lesiones más habituales y significativas que afectan a músculos, tendones y sistema esquelético.

\section{Introducción}

La práctica habitual de ejercicio físico o de algún deporte (actividad física reglada) a una intensidad moderada o alta confiere beneficios claros y objetivables en diferentes indicadores de salud. Sin embargo, hay que tener en cuenta que dicha práctica no está exenta del riesgo de padecer algún que otro tipo de lesión deportiva. Hoy en día no resulta extraño el oír hablar de alguien aquejado de «codo de tenista o de golfista», «hombro de nadador» o "pulgar del esquiador»; patologías todas ellas, que sin ser exclusivas de aquellos que practican el deporte del que toman el nombre, sí aparecen con mayor frecuencia entre sus practicantes. En las sociedades llamadas desarrolladas se ha incrementado notablemente la realización de actividad física o deporte du- 
rante las tres últimas décadas, coincidiendo en gran medida con la disminución de los requerimientos físicos de la mayoría de las ocupaciones laborales y el aumento del tiempo de ocio. Esta situación ha traído consigo la aparición de numerosas lesiones, fundamentalmente del sistema músculo esquelético, que pueden llegar a producir graves secuelas para el afectado, junto a un elevado coste económico y social. Por este motivo no debe extrañar que cada vez exista un mayor número de médicos del deporte, traumatólogos, rehabilitadores y fisioterapeútas que desarrollen su actividad profesional en este campo.

Entre los primeros relatos que recogen la presencia de lesiones deportivas se encuentran los textos clásicos que narran los Juegos Olímpicos (JJ.OO.) de la antigüedad. Cabe destacar el caso de Arrichion de Phigaleia, vencedor en el año $564 \mathrm{AC}$ en la prueba denominada pankration (mezcla de lucha y boxeo), tras lograr dislocar el tobillo de su oponente, un luchador procedente de Arcadia, y morir posteriormente mientras era declarado vencedor del combate. Se trata quizás del primer vencedor a título póstumo en unos JJ.OO. Quizá sea mas conocida la gesta bélica del emisario Filipides, que falleció en el Ágora de Atenas, tras retornar corriendo desde las llanuras de Maratón, y que posteriormente se recordaría en los anales del olimpismo como origen de la mítica carrera de maratón, una de las pruebas mas carismáticas del programa olímpico. En la reciente historia de los JJ.OO. de la era moderna, y mas concretamente tras las citas de Tokio y México que impulsaron las retransmisión deportivas por televisión a nivel mundial, son numerosas las imágenes de deportistas de renombre de ambos sexos que sufrieron importantes lesiones deportivas durante las pruebas en las que participaban, así como las gestas de aquellos otros deportistas, que aún estando lesionados, lograron sobreponerse y alcanzar resultados meritorios e incluso ser campeones olímpicos. A continuación se recogen algunas de estas gestas.

En los JJ.OO. de 1904 el gimnasta estadounidense George Eyser fue capaz de ganar 6 medallas ( 3 de ellas de oro) junto a once campeonatos nacionales compitiendo con una "pata de palo» consecuencia de un accidente ferroviario que le supuso la amputación de una pierna. Casi un siglo después cabe destacar a la también gimnasta estadounidense Kerry Strug que llevó a su equipo a conseguir la medalla de oro en los JJ.OO. de Atlanta en 1996, a pesar de competir en la final con una grave lesión de tobillo producida en las rondas clasificatorias. En la retina de todos los espectadores quedó grabada la imágen del famoso entrenador del equipo americano Karoly (valedor de la famosa gimnasta rumana Nadia Comanecci) llevando en brazos a su pupila 


\section{Lesiones en el deporte}

a recoger su medalla, en un acto que se entendió como la exaltación del sacrificio y afán de superación de los grandes deportistas, pero que desde el punto de vista médico nunca debería haberse consentido. Algo similar le ocurrió al famoso púgil Joe Frazier que logró la medalla de oro en los JJ.OO. de Tokio boxeando con un pulgar fracturado en las semifinales previas. Ante las advertencias de los médicos que le desaconsejaron tomar parte en la final, él alegó que tenía una mujer $\mathrm{y}$ tres hijos que alimentar de regreso a su tierra natal en Carolina del Sur.

Al Oerter, el mas famoso lanzador de disco de la historia, ganó en Tokio la tercera medalla de oro consecutiva en unos JJ.OO. (de las cuatro que consiguió) a pesar de padecer una lesión cervical y una fractura costal que le producían un intenso dolor en cada uno de sus lanzamientos.

El mejor saltador de trampolín que se recuerda, Greg Louganis, logró dos medallas olímpicas en los JJ.OO. de Seúl de 1988, y no dudó para conseguir la segunda en plataforma en ejecutar en su último intento el denominado "salto de la muerte», a pesar de competir con la cabeza suturada fruto de un fuerte traumatismo acaecido en la prueba de palanca (que también ganó) celebrada unos días antes.

Silken Laumann, remera canadiense campeona del mundo en 1991, fue embestida por la proa de otra embarcación apenas dos meses antes de la celebración de los JJ.OO. de Barcelona. El impacto le produjo una fractura abierta de la tibia junto a una parálisis peroneal, que la llevaron cinco veces al quirófano en el plazo de un mes. A pesar de ello, su tesón fue determinante en la consecución de la medalla de bronce en su prueba, apenas 78 días después de sufrir tan dramático accidente.

En España, y aunque fuera del deporte olímpico, podemos citar el reciente caso del vigente campeón mundial de motociclismo en la categoría de 500 c.c. Alex Crivillé que logró dicho campeonato compitiendo en las dos últimas pruebas del circuito con una fractura no reducida en su muñeca, fruto de una caída en una carrera previa.

Los infortunios deportivos reseñados se encuentren entre los citados con mayor frecuencia en la historia olímpica, y del deporte en general, por tratarse de deportistas de gran renombre. No obstante, con cierta frecuencia se producen episodios similares entre los miles de practicantes mas o menos anónimos de todo el mundo, lo que nos da una idea de la gran tolerancia al sufrimiento, junto a la enorme capacidad de recuperación de que hacen gala la mayoría de los deportistas amateurs o profesionales. 
A continuación se analiza en mayor profundidad aspectos mas concretos de las lesiones deportivas incluyendo la definición de las mismas junto a las principales causas que pueden influir en su génesis. Finalmente se analizan brevemente algunas de las lesiones mas frecuentes que se producen con la práctica deportiva.

\section{Concepto y epidemiología de la lesión deportiva}

¿Qué se entiende por lesión deportiva?

En EE.UU. se calcula que se producen entre 3 y 5 millones de lesiones deportivas anuales, lo que representa una de las primeras causas de lesión accidental entre adolescentes y adultos jóvenes. Para poder establecer medidas preventivas adecuadas, así como para tratar de evitar las posibles secuelas de toda índole que pudieran derivarse de las mismas, se hace necesario el precisar adecuadamente lo que se entiende por lesión deportiva. De forma simplista, y no por ello menos acertada, podría definirse como lesión deportiva a toda aquella que se produce mientras se realiza deporte o actividad física. No obstante, resulta necesario el establecer los criterios «mínimos» que permitan diferenciar una simple molestia física de una lesión ya establecida. Entre las distintas definiciones propuestas, cabe destacar las empleadas por el National Athletic Injury Registration System (NAIRS) en EE.UU., y ya en nuestro entorno, la enunciada por el Consejo de Europa (tabla 1).

TABLA 1. Concepto de lesión deportiva (L.D.)

1. (NAIRS) National Athletic Injury Registration System - EE.UU.: Aquella que impide la participación deportiva (entrenamiento y/o competición) al menos en el día después de haberse producido (Vinger, 1981)

- L.D. leve: incapacita entre 1-7 días

- L.D. mderada: incapacita entre 8-21 días

- L.D. grave: incapacita más de 21 días o produce secuelas permanentes

2. CONSEJO DE EUROPA: Cualquier lesión producida al tomar parte en un deporte que conlleve una o más de las siguientes consecuencias

- Reducción en la cuantía o nivel ed la actividad deportiva

- Requiere consejo (médico / de otra índole) o tratamiento

- Produce efectos sociales o económicos adversos 


\section{Lesiones en el deporte}

A tenor de las dos definiciones propuestas, cabe intuir que un gran número de lesiones que afectan a deportistas recreacionales (por motivos de ocio y/o salud) e incluso aficionados (o amateurs) no llegan ni a los hospitales ni a los ambulatorios, ya que son atendidas por los propios afectados o por allegados suyos, no siempre del entorno sanitario. Por este motivo resulta extremadamente complejo el disponer de estadísticas fiables acerca del número real de lesiones achacables a cada deporte (incidencia) o actividad física concreta.

\section{Epidemiología de las lesiones deportivas}

Como se ha mencionado previamente resulta complejo el conocer el riesgo lesional (siniestrabilidad) inherente a cada deporte, especialmente en el deporte no profesionalizado. En el deporte amateur federado se dispone al menos de la información recogida en los partes de lesiones que tramitan los deportistas accidentados a las compañías de seguros concertadas por cada federación. No obstante, la gran mayoría de lesiones que afectan a la población general cuando realiza actividad física en sus modalidades mas populares (andar, correr «jogging o footing», nadar, montar en bicicleta, gimnasia de mantenimiento-aerobic, o los diferentes deportes de raqueta) resulta casi imposible el poder contabilizarlas, exceptuando aquellos casos (generalmente los mas graves) que acuden a centros hospitalarios. A pesar de todas las limitaciones expuestas, los epidemiólogos intentan en sus estudios el cuantificar tanto el número de lesiones (incidencia) como el riesgo lesional (siniestrabilidad), empleando para ello el concepto de exposición, que se refiere a las horas de práctica deportiva en las que el sujeto es susceptible de padecer algún tipo de lesión. Este parámetro es fundamental tenerlo en cuenta antes de concluir que un determinado deporte entraña mas o menos riesgo de lesiones deportivas que otro, ya que la valoración del riesgo dependerá del tiempo total de exposición al deporte por la totalidad de practicantes y no del número de ellos.

\section{¿Por qué se producen las lesiones deportivas?}

Las lesiones deportivas pueden producirse por diferentes mecanismos (tabla 2) que en ocasiones pueden asociarse varios de ellos a la vez. 
TABLA 2. Mecanismos de producción de las lesiones deportivas

1. CONTACTO / IMPACTO

2. USO EXCESIVO (OVERUSE)

3. SOBRECARGA EXCESIVA (OVERLOAD)

4. VULNERABILIDAD EXCESIVA

5. POBRE FLEXIBILIDAD Y/O BALANCE MUSCULAR ADECUADO

6. CRECIMIENTO SOMÁTICO (NIÑOS Y ADOLESCENTES)

Existen a su vez diversos factores que se invocan como causantes directos, o favorecedores de la aparición de cada tipo de lesión deportiva. Estos factores suelen dividirse de forma genérica (tabla 3 ) en internos (aquellos inherentes al propio sujeto que realiza deporte) y externos (aquellos achacables al deporte y al entorno en que este se practica).

TABLA 3. Factores etiológicos de las lesiones deportivas

\section{INTERNOS}

1. EXISTENCIA DE UN DEFECTO / ANOMALÍA FÍSICA

2. CONDICIÓN FÍSICA DEL SUJETO

(resistencia aeróbica / fuerza / flexibilidad / velocidad / destreza deportiva-coordinación)

3. PRESENCIA DE LESIONES PREVIAS

4. PSICOLÓGICOS (autoestima / asunción riesgos / personalidad)

5. CONSTITUCIONALES (talla / peso / movilidad articular / adiposidad)

6. $\mathrm{EDAD} / \mathrm{SEXO}$

EXTERNOS

1. DEPORTE (modalidad y especialidad / exposición ( $\mathrm{n}^{\circ}$ horas práctica) / oponente)

2. INSTALACIONES (pavimentos / iluminación / medidas de seguridad)

3. EQUIPACIÓN (material / equipo protección / vestimenta)

4. AMBIENTALES (temperatura / humedad / viento)

5. ENTRENADOR-JUECES (reglamentos/ aplicación de los mismos)

\section{Lesiones musculares}

El músculo esquelético constituye la mayor masa de tejido en el cuerpo humano. En deportistas de competición y alto nivel constituye el $47,5 \%$ del total del peso del cuerpo en varones, llegando en algunas especialidades deportivas al 50\% y en mujeres la media es de un $45,6 \%$ obteniéndose incluso valores del $48,5 \%{ }^{1}$. 
El músculo esquelético consta de fibras musculares individuales, una red organizada de nervios y vasos sanguíneos, y una matriz de tejido conectivo extracelular.

El músculo, por el hecho de ser un tejido muy especializado, posee una alta sensibilidad frente a las lesiones, tanto mecánicas como isquémicas, de la misma forma que moderadas alteraciones funcionales podrían conducir a su atrofia.

Por otra parte y también atendiendo a sus características como tejido muy diferenciado, es incapaz de regenerarse de forma completa, de tal manera que las reparaciones de las pérdidas de sustancias o soluciones de continuidad se resuelven gracias al desarrollo de una cicatriz conjuntiva.

La notable vascularización del músculo y sus amplios espacios intersticiales explican la facilidad y extensión con que tienen lugar los fenómenos de edema, que a su vez, y de persistir en el tiempo, se organizan con facilidad ocasionando fibrosis y retracción muscular.

Por todas estas razones no sólo es importante la patología traumática muscular, sino también sus frecuentes y graves secuelas.

Las lesiones más frecuentes del músculo esquelético durante la práctica deportiva son la contusión y la rotura muscular, y mucho menos frecuente la laceración y la isquemia secundaria a síndrome compartimental.

\section{Contusión muscular}

Es consecuencia de un traumatismo cerrado y directo que, dependiendo de su intensidad, puede ocasionar desde pequeñas lesiones fibrilares, hasta necrosis de los haces musculares.

Los signos característicos son la presencia de equímosis y hematoma, que suele extenderse más allá del foco de contusión. La zona muscular afectada aparece infiltrada de sangre con coágulos interfasciculares y ocupando los intersticios fibrilares, en caso de existir. Tanto el grado de hemorragia local como las lesiones fibrilares dependen, en gran medida, del estado en el que se encontraba el músculo en el momento de producirse el traumatismo, y así, a igualdad de intensidad, son más graves las lesiones producidas sobre un músculo en contracción.

Las contusiones son más frecuentes en deportes de contacto como fútbol, rugby, hockey y en deportes de combate, judo, boxeo.... 
Una contusión, habitualmente, se distingue de la rotura muscular no sólo por el mecanismo de producción sino fundamentalmente, porque no suele producir impotencia funcional.

Los músculos más afectados son el cuádriceps y los gemelos aunque en la práctica deportiva pueden verse afectados otros grupos musculares tanto de la extremidad superior como de la inferior.

La contusión se manifiesta por dolor localizado, moderada impotencia funcional y equímosis posterior, en la mayoría de los casos. En los traumatismos de mayor intensidad pueden observarse hematomas crepitantes con fluctuación, impotencia funcional importante e incluso rotura del músculo.

El tratamiento va dirigido al control del edema, el hematoma y el dolor. Inicialmente con la aplicación de hielo en una bolsa sobre la zona afectada, una moderada compresión y elevación de la extremidad. La aplicación intermitente de hielo debe seguirse por 24-48 horas después de la intensidad del traumatismo. Está indicada la administración de antiinflamatorios y enzimas proteolíticas por vía general y local. La segunda fase, en cuanto el edema y la inflamación se han estabilizado, debe dirigirse a la restauración total de la movilidad ejercicios de extensión y flexión deben ser iniciados, teniendo en cuenta, no obstante, que estiramientos pasivos excesivos pueden favorecer la aparición de miositis osificante traumática.

\section{Roturas musculares}

En las roturas musculares el agente causal no es un traumatismo extrínseco del músculo, sino la propia contracción muscular de intensidad o coordinación inadecuada.

Las diferentes denominaciones o términos empleados para designar la lesión muscular como «contractura» «tirón», "roturas fibrilares» y «roturas musculares», no son sino, diferentes grados de lesiones (fibrilares, fasciculares, totales) de la fibra muscular.

En general las roturas son parciales, afectando con más intensidad la retracción a las fibras periféricas. Con mucha menor frecuencia, la rotura puede ser total, quedando ambos cabos musculares separados uno de otro, con una superficie de interrupción irregulares, retracción de los cabos y originando un gran hematoma. Las roturas fibrilares son las más frecuentes en el deporte y se producen en situaciones de alta velocidad como esprint o saltos. Los atletas refieren una sensación 
de dolor súbito e intenso en la zona muscular afectada e incluso algunos describen un chasquido coincidiendo con el estiramiento de dicha zona.

En la exploración física encontraremos fundamentalmente dolor e impotencia funcional; en la palpación podemos detectar un hematoma central y a ambos lados del mismo el músculo retraído, que se manifiesta como una masa blanda que aumenta de tamaño y consistencia con la contracción muscular. La separación que queda entre los extremos del músculo roto, sólo puede apreciarse si el músculo es superficial, e incluso así, puede quedar parcialmente cubierto por el hematoma existente.

Todos estos datos son más difíciles de explorar en las roturas parciales dado su menor tamaño y su situación muchas veces profunda, por eso si a la palpación observamos este tipo de defecto, debemos sospechar un tipo de lesión más severa con rotura muscular completa.

El alcance de la exploración debe estar guiado fundamentalmente por el dolor, no obstante, es importante valorar la afectación neurovascular. En muchos casos el atleta es incapaz de seguir compitiendo.

Las lesiones pueden ir acompañadas de una cantidad importante de sangrado, aunque frecuentemente se tardan días en poder detectar una equímosis subcutánea. El sangrado no queda confinado al músculo propiamente dicho, sino que puede escapara a través del perimisio y la fascia hasta el espacio subcutáneo.

Esto explica el hecho de que la equimosis observada aparezca, en ocasiones, distal al lugar de la lesión, a diferencia de las contusiones musculares, donde la equimosis queda confinada al mismo lugar de la contusión.

Por ser la lesión muscular alta o totalmente incapacitante para la práctica deportiva es necesario una vez realizado el diagnóstico clínico, establecer un diagnóstico más preciso que delimite la intensidad de la zona lesionada y la posible patología en estructuras adyacentes.

Dos son las técnicas de imagen que nos permiten obtener estos resultados:

- Ecografía muscular

- Resonancia Magnética (RM).

Consideramos que la técnica de elección es la ecografía de alta resolución que permite detectar lesiones de hasta 1-2 $\mathrm{mm}$, utilizando transductores de frecuencia variable entre $5-12 \mathrm{mHz}$.

Por la facilidad, inocuidad y la posibilidad de realizar exploraciones funcionales en tiempo real tanto con el músculo en contracción como en relajación, consideramos que es la técnica de elección para la mayoría de los casos. 
La RM, tan profusamente utilizada en la población deportiva, creemos que en la lesión muscular debería estar reservada a grupos musculares muy profundos como ocurre en la pelvis, o de compleja anatomía, como el manguito de los rotadores del hombro por presentar una mejor definición de la estructura anatómica que la ecografía.

En líneas generales el tratamiento inicial debe ir orientado hacia el control del dolor y de la inflamación. Así pues, la indicación fundamental, es la aplicación local de hielo y la compresión moderada del área afectada. Con los mismos objetivos, se han de hacer estiramientos pasivos en la medida en que vayan siendo tolerados.

La capacidad contractil del músculo puede verse reducida en un $50 \%$. Por ello durante la fase aguda deben evitarse las contracciones excéntricas del músculo, así como las inmovilizaciones en la medida de lo posible.

Los estiramientos concéntricos e isométricos se irán añadiendo según vayan siendo tolerados por el deportista incrementando la resistencia de forma progresiva. Los ejercicios isocinéticos tienen una especial utilidad porque permiten al atleta realizar los movimientos a un nivel adecuado con el que se sienta cómodo.

El programa de rehabilitación debe ir dirigida a restablecer el movimiento que debe alcanzar el mismo nivel que la zona no lesionada.

En cuanto al tratamiento quirúrgico, sólo en los casos de roturas completas del vientre muscular, con gran retracción de cabos y/o con un gran hematoma, es necesario la aplicación de dicho tratamiento en fase aguda.

De no ser así, existe un riesgo de resultado funcional mediocre y de las secuelas clásicas de fibrosis, cicatrices dolores y osificaciones.

\section{Síndrome compartimental crónico.}

El síndrome compartimental es un conflicto entre continente - fasciay contenido - músculo-. Cuándo la presión de un compartimento supera la capacidad de distensibilidad de la fascia que le rodea se produce el síndrome compartimental.

Aunque se puede distinguir una forma aguda y una crónica, es esta última la que con mayor frecuencia se da durante el ejercicio.

El ejercicio favorece la aparición de este síndrome debido al uso intensivo de los músculos, que produce hipertrofia muscular y por tanto aumenta la presión del compartimento, al aumentar el contenido del mismo. 
Las contusiones y lesiones de tejidos blandos favorecen la aparición del síndrome compartimental. La utilización de ortesis (rodilleras , musleras, o vendajes elásticos), que producen una compresión externa, no es probable que pudieran inducir el síndrome por sí solas, pero si favorece su aparición en algunas ocasiones en las que existe patología previa, puesto que disminuye el flujo muscular durante el ejercicio y el músculo se nutre mal.

En el síndrome compartimental crónico inducido por el ejercicio, son los músculos de la pierna los que son ejercitados con mayor intensidad en casi todos los deportes y por lo tanto, es la localización mas frecuente de síndrome compartimental. Los compartimentos más afectados de la pierna son el anterior y el lateral y con menor probabilidad, los posteriores superficial y profundo. En el miembro superior, son deltóides y bíceps donde habitualmente puede presentarse un cuadro de síndrome compartimental; generalmente en deportistas que practican remo, piragüismo, o lanzamientos.

Durante el ejercicio la contracción isométrica o isotónica intensa y repetida, eleva la presión intracompartimental lo que produce isquemia muscular mientras dura la contracción.

Otra circunstancia es que durante el ejercicio prolongado e intenso el volumen muscular se incrementa. Esta hipertrofia puede reflejar un aumento de permeabilidad capilar que ocasiona una acumulación de fluido en los espacios intra y extracelulares del músculo. El tercer hecho a considerar es que la hipertrofia de las fibras musculares provocada por el entrenamiento, dificulte el retorno venoso y linfático, asociado,en algunas ocasiones a pequeñas hemorragias por desgarros fibrilares, que serán las responsables del aumento del volumen muscular.

En cuanto a la clínica cabe destacar como síntoma principal la aparición de dolor sobre determinado compartimento al realizar el ejercicio. En un principio el dolor es moderado, de tal forma que el deportista es capaz de seguir realizando el ejercicio, aunque con el paso del tiempo el dolor se intensifica, le hace disminuir la intensidad del ejercicio y al final le obliga a parar. Es típica la desaparición del dolor con el reposo y la reaparición del mismo al volver a realizar el ejercicio. Esto explica que el síndrome compartimental aparezca cuando se inicia el entrenamiento mas intenso o después de una temporada de reposo.

El diagnostico de esta patología se realiza mediante la clínica y la confirmación de una elevación de la presión en el compartimento afectado. Esta presión debe superar los $15 \mathrm{mmHg}$ en reposo, los $30 \mathrm{mmHg}$ al minuto postejercicio y los $20 \mathrm{mmHg}$ a los 5 minutos postejercicio. 
En cuanto a la conducta a seguir en la forma crónica o recurrente del síndrome compartimental, no existe un acuerdo total. No obstante la indicación fundamental es la eliminación de los factores de riesgo. Para ello habrá que evitar los cambios bruscos en la intensidad del entrenamiento, así como procurar el comienzo progresivo después de largas temporadas de reposo y realizar un adecuado programa de musculación. Si todo esto no es suficiente, y el deportista desea volver al nivel de entrenamiento anterior, se ha de valorar la posibilidad de una fasciotomía.

La fasciotomía permite volver a la práctica del deporte de alto nivel a más del $60 \%$ de los deportistas operados. Los casos menos favorables que si bien han permitido la desaparición de las molestias, no han conseguido recuperar el nivel anterior a la lesión, se han atribuido a la reducción de la capacidad de mantener la tensión y presión muscular que se produce después de la fasciotomia.

\section{Tendinopatía por sobrecarga}

En medicina del deporte, las tendinopatías por sobrecarga representan un tipo de patología de especial relevancia. De hecho, se trata de cuadros patológicos muy frecuentes que típicamente afectan a las zonas anatómicas relacionadas con la modalidad deportiva practicada. Este tipo de lesiones, generan una sintomatología que limita o incluso impide de forma absoluta el desarrollo de la práctica deportiva. Además, si las tendinopatías no son diagnosticadas y tratadas correctamente, tienden a cronificarse y a recidivar.

Antes de profundizar en el tema, conviene apuntar algunos conceptos relativos a la morfología y la función del tendón normal.

Los tendones son estructuras anatómicas interpuestos entre los músculos y los huesos. Tienen como función transmitir las tracciones mecánicas de la contracción muscular a la palanca esquelética. El tejido tendinoso esta formado por unas células específicas denominadas tenocitos, y por la matriz fundamental, constituida a su vez, por fibras de colágeno y por algunas fibras elásticas. Sus propiedades mecánicas se deben principalmente a las fibras de colágeno. Estas fibras están organizadas en haces de primero y segundo orden, y se orientan en la dirección de aplicación de las solicitaciones mecánicas a las que se somete.

Las fibras de colágeno están constituidas por fibrillas que a su vez, resultan de la unión de muchas microfibrillas. 


\section{Lesiones en el deporte}

El colágeno del tendón es del tipo genético I, que es el adecuado para soportar la tensión a la que esta sometido durante la contracción muscular. Por eso, esta estructura representa un ejemplo perfecto de adaptación estructural a una función determinada. A pesar de esta superespecialización, el tendón puede verse envuelto en lesiones por sobrecarga y esto es debido a que en casi todas las prácticas deportivas se requieren actuaciones del aparato locomotor, llevadas al límite de lo fisiológico.

En la cadena estructural, constituida por músculo-tendón-huesoarticulación, el tendón es, sin duda, la parte más débil. El músculo por su parte, tiene la capacidad de adaptarse fácilmente a la sobrecarga funcional, mediante la hipertrofia del propio músculo, de tal forma que, mediante técnicas adecuadas, el atleta puede potenciar notablemente su masa muscular. El tendón por el contrario no tiene esta capacidad de adaptación, por lo que con mayor frecuencia puede verse afectado por lesiones de fatiga.

Esto no quiere decir que cualquier método de potenciamiento muscular provoque irremediablemente una tendinopatia, sino que, la sobrecarga funcional del aparato locomotor, provocada por el deporte, es un factor de riesgo para la integridad de los tendones.

En cuanto a la etiología de las tendinopatías, podemos hablar de factores intrínsecos y extrínsecos.

Dentro de este grupo cabe destacar los trastornos de las condiciones de salud del atleta, así como de la condiciones psicológicas que actúan sobre su concentración y su coordinación motora. Una coordinación imperfecta entre el músculo agonista y antagonista provoca un aumento de la tensión intensa durante la contracción y una ejecución inadecuada del gesto deportivo. De especial relevancia, y frecuentemente infravalorado, son el calentamiento muscular mal efectuado antes de la competición, y la deficiente ejecución de los ejercicios de estiramiento y flexibilidad muscular. De hecho, esto último, puede ser un método útil en la profilaxis de las tendinopatías. De tal forma que la distensión de los músculos agonistas y antagonistas mejora la elasticidad y la coordinación muscular y reduce, por ello, las tensiones mecánicas que se ejercen sobre el tendón.

En este grupo de factores extrínsecos deben añadirse también, las condiciones climáticas, los métodos de entrenamiento y potenciación muscular incorrectos, y los materiales, ( calzado, equipo, terreno), inadecuados.

En particular los terrenos duros o los muy rápidos provocan ondas mecánicas de mayor intensidad y consecuentemente más lesiones para el tendón. 
Analizados todos estos factores, estamos en disposición de afirmar que, la solución del problema de las tendinopatías por sobrecarga funcional, radica fundamentalmente, en la profilaxis, mediante el control minucioso de todos estos factores tanto extrínsecos como intrínsecos.

No obstante hoy en día se pueden contar con numerosos procedimientos terapéuticos en caso de que fueran necesarios.

Las tendinopatías que se presentan en deporte con mayor frecuencia son:

a. Miembro superior: - Tendinopatía del manguito de los rotadores.

- Epicondilitis.

b. Pelvis: - Síndrome de la inserción de los aductores.

- Síndrome de la inserción de los isquiosurales

c. Miembro inferior: - Tendinopatía aquilea.

- Tendinopatía rotuliana.

La clínica de las tendinopatías, se caracteriza invariablemente por dolor e impotencia funcional.

El dolor tendrá una localización y una evolución en relación directa con la biomecánica del gesto deportivo realizado.

En la exploración física encontraremos signos objetivos, como un aumento del volumen del tendón, en algunos focos, dolor constante por presión sobre la zona afectada y por las maniobras que lo desplazan mecánicamente (distensión pasiva o contracción) contra una resistencia.

Como ya hemos dicho anteriormente la sintomatología es característica de cada zona anatómica.

En la tendinopatía del manguito de los rotadores, el dolor se localiza en el hombro, en el punto de inserción muscular, es decir, bajo el acromión y a la altura del troquiter. El dolor aumenta en los movimientos de abducción, rotación externa y retropulsión.

En la epicondilitis o "codo de tenista», el dolor se localiza en el codo, a nivel de la inserción de los músculos extensores de la muñeca. La presión, el estiramiento pasivo o contrarresistencia, provoca un dolor agudo en dicha zona.

La tendinopatía de aductores, propia de futbolistas y jugadores de rugby, es un trastorno tendinoso insercional, de los músculos aductores de la cadera y del músculo recto del abdomen. En este caso el dolor se localiza en la región inguinal y aumenta con los movimientos de aducción de la cadera, contracción de la musculatura abdominal, así como por presión sobre la zona de inserción púbica de los grupos musculares anteriormente citados. 
En la tendinopatía del semimembranoso, semitendinoso y bíceps femoral, el dolor se localiza en la zona isquiática en le momento de acelerar o alargar el paso.

En la tendinopatía del rotuliano hay que diferenciar una patología de inserción proximal del tendón, a nivel del polo inferior de la rótula, y-una patología de inserción distal a nivel de la tuberosidad tibial anterior. El atleta refiere clínica dolorosa sobre la cara anterior de la rodilla en la región rotuliana. Es el dolor a la presión el que nos orienta sobre una patología en la zona de inserción proximal o distal del tendón. Además en las lesiones crónicas se observa un aumento del volumen del tendón en el que se puede palpar pequeños nódulos.

En la tendinopatía aquílea se reconocen tres formas clínicas diferenciadas.

Las tendionapatías de desarrollo hacia una peritendinitis, hacia una tendinosis y la tendinopatía insercional. La sintomatología subjetiva queda definida por dolor en la región aquilea que aparece al caminar especialmente al llevar calzado con tacón bajo, y durante la carrera, especialmente en terreno accidentado, y que tiende a atenuarse tras un calentamiento adecuado.

En la exploración física es fácil distinguir las tendinopatías insercionales, las cuales se caracterizan por una tumefacción de la región del calcáneo, especialmente si se asocia a una bursitis preaquílea, y por dolor a la presión sobre la zona de inserción del tendón al hueso, o delante del tendón, en donde se halla la bolsa serosa. Sin embargo resulta más difícil, desde el punto de vista clínico, distinguir una peritendinitis, lesión de carácter preferentemente inflamatorio, de pronóstico relativamente favorable, de una tendinosis, lesión típicamente degenerativa, con pronóstico menos favorable. En ambos casos se produce una tumefacción, con aumento del volumen del tendón, y se desencadena dolor a la presión. En las formas más graves de tendinosis se puede palpar en el tendón pequeñas formaciones nodulares que presentan áreas de degeneración del tejido tendinoso.

El diagnóstico de las tendinopatías, además de por sus características clínicas, se basa, al igual que las lesiones musculares, en dos técnicas fundamentales como son la ecografía musculotendinosa y la RM, que además de su función diagnóstica tiene utilidad en la valoración pronostica y la elección terapéutica.

El tratamiento de las tendinopatías del deporte puede orientarse hacia un tratamiento conservador o quirúrgico. 
El tratamiento conservador tiene sus indicaciones precisas en las fases iniciales, si éste se aplica oportuna y correctamente produce la curación en un elevado porcentaje de casos.

El esquema terapéutico conservador es el siguiente:

a) Reposo activo: eliminación de agresiones mecánicas de la unidad musculotendinosa afectada, pero con adecuado entrenamiento y potenciación de las restantes parcelas musculares.

b) Farmacológico: AINES y antiedematosos por vía oral o local en preparaciones en gel o crema. Las infiltraciones en general quedan limitadas a las tendinopatías insercionales con bursitis.

c) Otros: crioterapia, iontoforesis con antiinflamatorios, láser, terapias con ultrasonidos... Protecciones ortopédicas.

Cuando la terapia conservadora no tiene éxito, por recidivas o cronificación, se recurre al tratamiento quirúrgico. No obstante esto no debe aplazarse excesivamente, de hecho el buen resultado del tratamiento quirúrgico depende de que la indicación sea correcta y el diagnóstico más detallado posible.

En cuanto a la rehabilitación postoperatoria, debe plantearse una movilización sin carga, lo más rápido posible y en algunos casos inmediata, del miembro operado.

\section{Columna vertebral}

De las numerosas patologías que pueden afectar a la columna vertebral, dos son los cuadros que tienen una clara mayor incidencia en deportistas, por ser la causa desencadenante la práctica deportiva intensa y repetida la espondilolisis y la epifisitis vertebral.

\section{Espondilolisis y espondilolistesis}

Espondilolisis es la existencia de un defecto a nivel de la pars interarticularis de una vértebra. Este defecto puede ser displásico con elongación y adelgazamiento de dicha pars, o ístmico cuando existe una solución de continuidad en la estructura. El nivel más frecuente es la quinta vértebra lumbar, menos frecuente la cuarta y tercera y excepcional a otros niveles.

Espondilolistesis es el desplazamiento de un cuerpo vertebral en relación a su inmediatamente inferior.

Las espondilolistesis se han clasificado en cinco tipos:

- Displásica 
- Ístmica

- Degenerativa

- Traumática

- Patológica

En la población deportiva, es el tipo II, espondilolistesis ístmica, la más frecuente. La lesión básica que permite la listesis está en la alteración ístmica. Las carillas asticulares mantienen su normal relación, mientras que la pars interarticularis está fracturada, por sobrecarga y muy raramente de forma aguda por un único y violento traumatismo.

Actualmente, la teoría etiopatogénica más aceptada es la que considera la espondilolisis como una fractura de sobregarga que asienta sobre un istmo vertebral predispuesto anatómica, biomecánica y genéticamente.

Existen tres subtipos:

Subtipo A - Lítica. Secundaria a una fractura por fatiga del istmo.

Subtipo B - Elongación ístmica. En este caso la fractura de sobrecarga consolida repetidas veces con lo cual el istmo se va alargando progresivamente.

Subtipo C - Fractura ístmica aguda. Secundaria a un trauma severo.

$\mathrm{Si}$ bien, el factor desencadenante es la fractura por sobrecarga, también existen factores predisponentes a considerar:

Raza. El factor racial, como factor predisponente de espondilolisis ha cambiado en los últimos años. La raza esquimal tradicionalmente presenta una mayor incidencia. Cuando esta población ha perdido, en parte, sus costumbres tribales y las grandes distancias se hacen en vehículos motorizados y no andando, y la pesca y la caza se realizan con menos intensidad o de diferente manera, la incidencia ha disminuido. Si bien el factor racial existe, el factor ambiental tiene una gran importancia en la aparición de espondilolisis en la raza esquimal.

Herencia. Se han establecido una mayor prevalencia y espondilolistesis en algunas familias.

Otro argumento que defiende el factor hereditario en el defecto ístmico son los hallazgos de la lesión en gemelos univitelinos.

Sexo. Las mujeres de raza negra son el grupo con menos prevalencia de espondilolisis (1.1\%) y los varones de raza blanca los que mayor número de lisis acumulan (6.4\%).

Edad. En los estudios embriológicos jamás se ha encontrado lesión ístmica, desmintiéndose así la teoría congénita de espondilolisis. La mayoría de trabajos observan la aparición de lesión ístmica a los 5-6 
años, aumentando muy discretamente hasta la adolescencia, momento a partir del cual no aumenta la incidencia.

El factor, por tanto, desencadenante de la espondilolisis es la sobrecarga que sufre la pars en la actividad deportiva. Se describen unos deportes en los cuales existe un estrés preferentemente axial, otros rotacional y por último aquellos que sufren un estrés por hiperextensión lumbar.

En el grupo I se encuentran los deportes sometidos a estrés por compresión. Estos son: halterofilia, atletismo (salto de altura, fondo y campo a través), fútbol americano, rugby, judo, hípica, voleibol...

En el grupo II se hallan los deportes en los que predomina la torsión: tenis, golf, béisbol, baloncesto, fútbol, boxeo, voleibol, piragüismo y lanzamiento de disco.

En el grupo III ser encuentran los deportes en los que predomina la hiperextensión: gimnasia, tenis, remo, atletismo (salto de altura, lanzamiento de jabalina) y natación (en especial mariposa y braza).

Un número muy significativo de deportistas, en nuestra experiencia he $\quad 2040 \%$ con espondilolisis y/o espondilolistesis permanecen as abitual que los períodos, sin clínica significativa, se dros de dolor que coinciden con períodos de mayor ca into o con molestias poco significativas.

Incrementan ei desarrollo de sintomatología severa a partir de una espondilolistesis, el deslizamiento mayor al 25\%, y degeneración discal.

Clínicamente el dolor secundario a la producción de una espondilolisis, se sitúa generalmente en la parte lumbar inferior y no tiene irradiaciones en extremidades inferiores.

El dolor aumenta con la mínima extensión lumbar. La sintomatología se inicia normalmente después de realizar una actividad física importante, especialmente si ésta es de flexoextensión repetida. Su duración será mayor en aquellos casos en los que el individuo continúe con ejercicio físico más o menos vigoroso.

El deportista más raramente puede sufrir dolor de tipo radicular en una o las dos extremidades inferiores asociada a dolor lumbar bajo.

El dolor radicular puede producirse por el acodamiento de la raíz sobre el canto prominente de la vértebra retrasada, o por la estenosis del foramen debida al nódulo fibrocartilaginosos que se produce en la pars.

Otro síntoma importante es la contractura de los isquiosurales.

La contractura o espasmo de los isquiosurales se observa en la mayoría de los casos sintomáticos y se considera consecuencia de una irritación de la raíz nerviosa. Los músculos isquisurales están inervados 
por diversas raíces nerviosas, que abarcan desde L4 a S3, por tanto, éstos podrán estar interesados sea cual sea el nivel de la lesión.

Los deportistas adolescentes con patología listésica desarrollan una actitud escoliótica lumbar. Esta actitud nunca será estructural ni superará los $15 \%$.

El avance de los métodos diagnósticos, radiología convencional y digital, tomografía axial computerizada y resonancia magnética, nos permiten realizar un diagnóstico precoz, preciso y exacto.

La conduca a seguir, lógicamente, es suprimir o atenuar el factor desencadenante, es decir, la sobrecarga axial, rotacional o por hiperextensión a que esté sometido el deportista. Esto exige conocer los gestos deportivos de cada uno de los deportes, para emitir un consejo médico.

Los obligados controles médicos cada 6 o 12 meses, durante la época de crecimiento y cada 1-2 años a partir de los 20 años, nos permitirán valorar la tolerancia y la intensidad de la práctica deportiva que puede soportar el deportista, sin riesgo para su salud, y cuando así no sea, aconsejar el abandono de la práctica deportiva temporalmente, y con menos frecuencia definitivamente.

\section{Epifisitis vertebral}

La epifisitis dorsal juvenil descrita por Scheuermann, es una distrofia asociada a deformidad cifótica dorsal, que tiene un patrón fisiopatológico, histológico y radiológico concretos, pero una etiología aún desconocida. Situada preferentemente en el raquis torácico, puede afectar al raquis toracolumbar. Se caracteriza por el acuñamiento, de por lo menos 5 grados, de tres vértebras torácicas como mínimo, la existencia de diversas hernias discales intraesponjosas -también conocidad como hernias de Schmörl-, la disminución de los espacios intervertebrales y la irregularidad de los platillos vertebrales torácicos.

Los denominados «nódulos de Schmörl» responden a fenómenos de sobrecarga mecánica y se sitúan en las últimas torácicas o especialmente, primeras lumbares, pero nunca interesan al anillo epifisario del cuerpo vertebral.

En la práctica deportiva existen unos casos que afectan al anillo epifisario y responden a microtraumatismos repetidos. Son las «epifisitis asépticas». Son lesiones de la unión discovertebral, en las cuales el dolor y la ausencia de clínica neurológica son los síntoimas básicos, originados por una sobrecarga selectiva y localizada. 
Estas alteraciones vertebrales se encuentran fundamentalmente en deportistas jóvenes sometidos a una importante exigencia física y afectan a una o dos vértebras de la transición tóracolumbar. Las epifisitis lumbares suelen ser pequeñas, aunque en deporte de alto nivel practicado por adolescentes, como es la gimnasia, existen casos muy severos.

La diferencia entre las dos entidades reside en que la hernia de Schmörl se afecta el platillo óseo central y en la epifisitis lumbar se afecta el anillo epifisario que lo rodea.

Son unos deportistas adolescentes, sometidos a una alta exigencia deportiva donde la mecánica de hiperextensión forzada es obligada. $\mathrm{El}$ gesto de hiperextensión en sesiones de entrenamiento de 5-6 horas diarias comporta una importante hiperpresión del núcleo pulposo sobre la parte anterior del anillo fibroso que se coloca a máxima tensión contra el ligamento vertebral común anterior, a nivel de la parte anterior y media del cuerpo vertebral. Esta hiperpresión anterior del disco, intensa y repetida, reperecute sobre el núcleo marginal en pleno desarrollo, distorsionándolo, desplazándolo y provocando alteraciones de crecimiento y fenómeno de osteonecrosis, evitando su fusión y provocando hernias intraesponjosas.

La clínica de esta patología microtraumática es poco expresiva, existe un moderado cuadro de dolor lumbar. La radiografía presenta una imagen lítica, en la proyección de perfil, en el ángulo superoanterior vertebral. Los casos más severos pueden evolucionar hacia la inestabilidad y discartrosis precoz, por lo que es recomendable disminuir la actividad física vigorosa y realizar un control clínico y $\mathrm{Rx}$ periódico, para comprobar la estabilización dèl raquis, antes de reanudar la práctica deportiva de alto nivel. Un seguimiento médico no adecuado puede condicionar un dolor lumbar crónico en la edad adulta.

\section{Fracturas de estrés}

\section{Introducción}

Las fracturas de estrés son fracturas parciales o completas del hueso como resultado de una remodelación ósea acelerada en respuesta a un microtraumatismo habitual no violento y repetitivo ${ }^{2}$.

En el terreno deportivo las fracturas de estrés varían en incidencia y localización dependiendo del deporte y tipo de actividad. Las de tibia y metatarsianas son más frecuentes en corredores las del astrágalo en baloncesto y la del $\mathrm{V}$ metatarsiano es más frecuente en jugadores 
de balonmano, voleibol y fútbol americano. Actividades como la carrera, la gimnasia, el aeróbic o el baloncesto, se asocian a una alta incidencia de fracturas de estrés.

\section{Etiología}

El hueso es un tejido dinámico que responde con cambios en relación a las variaciones de intensidad o frecuencia de carga que se aplican. En general el hueso se adapta bien a las variaciones graduales de tensión. Cuando se aplican diferentes cargas, el hueso se remodela de forma gradual depositando hueso donde la tensión es mayor y reabsorbiéndolo donde no existe carga.

La fractura de estrés se produce cuando la tensión ósea producida por cargas repetidas sobrepasan la capacidad de resistencia del hueso. $\mathrm{Al}$ aumento de la carga se añade otro factor: la fatiga inducida por el ejercicio repetitivo disminuye la función de absorción de los músculos, por lo que se transmite una mayor tensión al hueso provocando la fractura. ósea.

El deportista gana fuerza muscular más rápidamente que fuerza

Una alta concentración de fuerzas musculares excéntricas y concéntricas actuando sobre un hueso específico predisponen al fallo óseo.

\section{Factores predisponentes}

- Actividad física: deportes de ejecución vigorosa y repetitiva asociados a carga física $\mathrm{y}$, en muchas ocasiones, aumento repentino en la intensidad, la frecuencia o la duración del ejercicio.

- Factores biomecánicos:

Torsión tibial y el grado de rotación externa de la cadera, asociado a calzado inadecuado y a superficies duras o irregulares, actuando de forma independiente y en conjunto.

- Factores hormonales y nutricionales:

Oligomenorrea, amenorrea primaria o secundaria, menarquia retardada, fase lútea alterada, ovulación alterada e infertilidad asociadas a actividades físicas intensas como la carrera, el aeróbic o la danza en mujeres con peso y porcentajes de grasas bajas.

En realidad, la causa primaria sería una concentración baja de estrógenos en sangre. 


\section{Diagnóstico}

El diagnóstico, a pesar del espectacular avance de los medios diagnósticos, sigue siendo eminentemente clínico. Una historia médico-deportiva en la que se refiera actividad física intensa y habitual, asociada a un dolor cada vez más intenso sobre una estructura ósea debe hacer sospechar siempre la existencia de una fractura de estrés en fase inicial.

El dolor tiene unas características muy definidas; inicialmente aparece al final del entrenamiento, afectando a una zona poco delimitada, para rápidamente hacerse persistente durante el entrenamiento y después de éste. Simultáneamente el dolor se hace selectivo con callo palpable e inflamación de partes blandas adyacentes.

La radiología convencional en los primeros días es poco específica, no visualizándose las alteraciones óseas en muchas ocasiones hasta las dos semanas de inicio de los síntomas. La gammagrafía ósea con tecnecio 99 permite detectar las alteraciones óseas precozmente y con una técnica en triple fase hacer el diagnóstico diferencial con lesiones de partes blandas, al ser positiva exclusivamente en la fase tardía en las fracturas de estrés. La tomografía axial estará indicada cuando estén afectadas partes anatómicas de morfología compleja como el astrágalo o el sacro. La resonancia magnética es, en el momento actual, el medio diagnóstico de elección al unir la precocidad en el diagnóstico con la especificidad y definición morfológica que no poseen otros medios diagnósticos.

\section{Tratamiento}

La base fundamental del tratamiento será de reposo activo. En el deportista de competición, por tanto, la limitación de la carga sobre el área afectada, acompañada de un programa de mantenimiento de función cardiovascular, fuerza muscular, flexibilidad y función propioceptiva,a permitirá una adecuada vuelta a los programas de entrenamiento una vez objetivada la consolidación ósea. Esta consolidación supone como mínimo de 4 a 7 semanas en función de la localización e intensidad de la lesión, pudiendo prolongarse hasta tres meses en algunos casos.

El entrenamiento en piscina o en bicicleta estática pueden ser útiles como elementos de trabajo alternativo.

El control de la evolución puede realizarse con radiología convencional y siempre será la ausencia de dolor la que marcará la reanudación de la actividad deportiva habitual. 


\section{Notas}

1 Los datos han sido obtenidos en el Servicio de Antropometría del Centro de Medicina del Deporte (C.S.D.), por la Dra. Alicia CANDA, sobre más de 5.000 deportistas de competición españoles de diferentes modalidades deportivas.

2 BRIETHAUPT, médico militar prusiano, fue el primero en describir, en 1850, las fracturas de estrés, en soldados tras largos y repetidos desplazamientos a pie, denominándolos "fracturas de marcha".

Después del descubrimiento de los Rayos X en 1896, fue una de las primeras lesiones diagnosticada y visualizada por radiografía.

La mecanización de los ejércitos y la intensificación de la práctica deportiva hace que sea en el ámbito deportivo donde actualmente se dan la mayoria de las fracturas de estrés en hueso sano. 